\title{
III SELBSTDARSTELLUNGEN DER KOOPERIERENDEN VEREINE
}

\section{Afghanischer Kulturverein AKIS (Afghanische KULTUR, INTEGRATION, SOLIDARITÄT)}

\section{Vorstellung des Vereins}

Der afghanische Kulturverein AKIS (Afghanische Kultur, Integration, Solidarität) besteht seit 26 Jahren und ist seit seiner Gründung mit der Problematik der afghanischen Geflüchteten in Europa befasst. Zunächst war es unser größtes Ziel, den Österreicher/ inne/n durch diverse Veranstaltungen die Kultur Afghanistans zu präsentieren mit der klaren Botschaft, dass nicht alle Afghan/inn/en zu den Taliban gehören. Mit der Zeit ist unser Verein bekannter geworden und unsere Schwerpunkte haben sich an die aktuellen Bedürfnisse der afghanischen und österreichischen Gesellschaft angepasst.

\section{Eine kurze Auflistung der Aktivitäten:}

- Geflüchtete in Griechenland: Letztes wichtiges Ereignis war der Brand im Flüchtlingslager von Moria. Der Obmann des Vereins AKIS flog nach Griechenland, um die Geflüchteten auf dem Festland vor Ort zu unterstützen. Seit Moria hat der Verein AKIS auch ein Netzwerk in Griechenland aufgebaut, um afghanischen Geflüchteten, die nicht in Moria leben, aber trotzdem Schwierigkeiten haben, $\mathrm{zu}$ helfen. Insgesamt ist der Verein in ganz Europa, in Afghanistan und den USA vernetzt.

- Unterstützung bei der Integration: Letztes großes Projekt war im Jahr 2017, dass ein Beratungszentrum für Geflüchtete aus Afghanistan in Wien gestartet wurde. Es wurden Beratungen, Deutschkurse, Workshops und Nachhilfe verständlich in den jeweiligen Muttersprachen angeboten. Obwohl das Projekt ein großer Erfolg war, haben wir seit der Amtszeit von Ex-Innenminister Herbert Kickl hierfür keine Unterstützung mehr erhalten.

- Beratung und Aufklärung: Ein wichtiges Medium ist die Frauenzeitschrift BANU. Wir klären Frauen unter anderem über ihre Möglichkeiten und Rechte in der neuen Heimat auf und motivieren sie, sich zu integrieren und aktiv an der österreichischen Gesellschaft teilzunehmen. 
- Engagement für Frauenrechte: Zuletzt hatten wir eine große Podiumsdiskussion in Zusammenarbeit mit VIDC mit dem Titel „Kein Frieden in Afghanistan ohne Frauen“. $\mathrm{Zu}$ Gast waren Vertreter/innen der afghanischen Regierung und der UNO. Insgesamt haben wir 2020 drei Workshops zum Thema „Gewalt gegen Frauen" in Zusammenarbeit mit Vereinen aus Österreich, Deutschland und den Niederlanden organisiert.

- Hilfe vor Ort: Jährlich fliegt der Obmann des Vereins AKIS, Herr Mir Ghous Uddin, nach Afghanistan, um dort vor Ort die Bevölkerung zu unterstützen. Der Verein fördert laufend drei Mädchenschulen bei der Behebung von eminenten Mängeln, nämlich der Anbringung von Sichtschutz auf dem Sportplatz (damit die Mädchen ungestört turnen können), bei der Einrichtung von externen Klassen (damit infolge Überfüllung der Klassen nicht in der prallen Sonne unterrichtet werden muss), beim Ankauf von Schulmöbeln, beim Zugang zu sauberem Trinkwasser etc. Außerdem wird stets besonderes Augenmerk auf die Unterstützung von Witwen und Waisenkindern gelegt.

Es gibt noch zahlreiche Punkte, die angeführt werden können, wie z.B. die bisher sechs Friedenskonferenzen in Wien, an denen auch der Sprecher des afghanischen Präsidenten und andere ranghohe Politiker Afghanistans teilnahmen, die Hilfe für verstorbene Angehörige afghanischer Geflüchteter in Österreich bei der Organisierung von Begräbnissen, die Unterstützung bei Familienstreitigkeiten, interkulturelle (Sport-) Veranstaltungen zum besseren Verständnis der Kulturen, Begleitung bei Behördenwegen etc. Näheres kann man auf unserer Homepage nachlesen: $<$ http://www.akiseu. com>.

Die kulturelle und sprachliche Orientierung des afghanischen Kulturvereins ist auf Afghanistan als Ganzes ausgerichtet, was innerhalb der afghanischen Community in Europa eine Seltenheit ist, da in Afghanistan viele verschiedene Volksgruppen leben. Die meisten gründen in der Emigration Vereine, die jeweils auf die Bedürfnisse der eigenen Volksgruppe ausgerichtet sind. Im Gegensatz dazu sind im afghanischen Kulturverein AKIS alle Volksgruppen der afghanischen Gesellschaft vertreten. Ausgeschlossen sind nur Personen, die extremistisches, fanatisches, faschistisches oder nationalistisches Gedankengut vertreten.

Der Verein hat mittlerweile ca. 200 Mitglieder in ganz Österreich. Jede/r kann dem Verein beitreten, ungeachtet seiner/ihrer Herkunft, Religion oder Sprache.

\section{Herausforderungen im COVID-19-Lockdown}

COVID-19 und der damit einhergehende Lockdown stellten für unseren Verein große Herausforderungen dar. Viele geplante Veranstaltungen mussten mehrmals verschoben oder abgesagt werden. Die persönlichen Beratungen und Kurse mussten 
ausgesetzt werden. Dafür wurden wir mit telefonischen Anfragen überhäuft. Der Verein hat Social Media und das Frauenmagazin BANU verstärkt genutzt, um die afghanische Community zu erreichen.

Eine erhebliche Herausforderung für den Verein manifestiert sich darin, dass viele Menschen in der afghanischen Community COVID-19 nicht ernst nehmen. Wir haben mit Kurzvideos sowie Informationstexten über die Gefahren des neuen Virus informiert und über die Maßnahmen der Regierung aufgeklärt. Das afghanische Frauenmagazin BANU haben wir genutzt, um unter anderem auf die Gefahren des Virus hinzuweisen, aber auch über Hilfe bei häuslicher Gewalt informiert. Leider verfügten wir nicht über die finanziellen Mittel, um das Magazin flächendeckend an alle afghanischen Geflüchteten sowie sozial schwachen afghanischen Familien in Österreich zu verteilen. Ein Hilferuf an das Bundeskanzleramt, das Gesundheits- und Innenministerium blieb leider unbeantwortet. Dabei ging es um machbare Unterstützung mit einer hohen Wichtigkeit. Wir gehen daher davon aus, dass die politisch Verantwortlichen zu sehr mit anderen Themen beschäftigt waren bzw. sind.

Die familiären Probleme innerhalb der afghanischen Community sind während der Lockdowns auch deutlich angestiegen. Der Verein hat viele Anrufe erhalten, um als Vermittler bei Eheproblemen oder sonstigen familiären Konflikten als vernünftige dritte Stimme schlichtend zu intervenieren. Vor allem Herr Mir Ghous Uddin, Obmann des Vereins AKIS, wird von vielen jungen Ehepaaren in der Diaspora als Vaterfigur angesehen. Wir nützen die Zeitschrift BANU, um Frauen zu unterstützen und sie auch über häusliche Gewalt aufzuklären. Es würde vielen Familien, vor allem den sozial schwachen, sehr helfen, die Auflage zu erhöhen und kostenlose Exemplare zu versenden. Hierfür fehlen uns jedoch leider die finanziellen Mittel.

\section{Appell an die Regierung}

Wir wissen, dass die Regierung derzeit angesichts der anhaltenden Coronaprobleme sehr überlastet ist. Wir würden uns dennoch wünschen, dass man in dieser Situation nicht auf die Vereine vergisst. Diese sind nämlich ein wichtiges Instrument, um tatsächlich alle in Österreich lebenden Afghaninnen und Afghanen effektiv zu erreichen.

Wir wären bereit, wichtige Informationen in unserer Frauenzeitschrift BANU zu übernehmen, die Auflage zu erhöhen und diese kostenlos an die afghanische Community und vor allem sozial schwache afghanische Familien zu verteilen.

Auch hätten wir die Möglichkeit, um z.B. Online-Workshops oder Seminare (z.B. zum Thema häusliche Gewalt) abzuhalten oder Beratung vor Ort in den Asylheimen verständlich in den Muttersprachen Dari, Pashto, Farsi, Hindi, Urdu, Russisch und Usbekisch durchzuführen und vieles mehr. Wir sind ein sehr aktiver und engagierter Verein, der viel umsetzen kann, jedoch fehlt es uns an Unterstützung durch die Regierung.

Außerdem würden wir gerne einen größeren Beitrag dazu leisten, um die Flücht- 
lingskrise einzudämmen. Wir würden gerne in Afghanistan Ausbildungswerkstätten aufbauen, damit die Jugendlichen dort einen Beruf erlernen, um sich in der Heimat eine Zukunft aufbauen zu können. Auch hierbei würden wir Unterstützung benötigen.

\section{Meinung über die Studie}

Wir sind sehr froh über die Durchführung der vom WWTF finanzierten Studie und bedanken uns bei Ihnen recht herzlich für Ihr Engagement. Leider wird oft vergessen, welchen Beitrag die Kulturvereine leisten, um für ein friedvolles Miteinander zu sorgen bzw. welche Schwierigkeiten die einzelnen Communities mit Migrations- bzw. Fluchthintergrund in Österreich haben, deren Sprachrohr eben die Kulturvereine sind, weil die Menschen in der Diaspora sich diesen anvertrauen. Dank dieser Studie konnten wir einen kleinen Einblick in das Engagement, aber auch in die Schwierigkeiten des afghanischen Kulturvereins AKIS gewähren. 


\section{AMAL - Initiative ZUR Begleitung von Migranten- FAMILIEN AUS DEM NAHEN OSTEN}

Eckdaten: Auftaktveranstaltung: November 2015, Vereinsgründung Jänner 2016, Mitgliederzahl: 650 Migrantinnen und Migranten, 80 Ehrenamtliche.

AMAL ist arabisch und heißt Hoffnung. AMAL begleitet Migrantinnen und Migranten aus dem Nahen Osten, vor allem aus Syrien und dem Irak, und hilft ihnen, in Österreich heimisch zu werden. Das Erlernen der deutschen Sprache und die persönliche zwischenmenschliche Beziehung, also das Kennenlernen und Ermöglichen von Freundschaften untereinander, sind dafür Schlüsselfaktoren. Das gelingt durch Sprachkonversationsrunden, in denen Ehrenamtliche integrationswillige Migrantinnen und Migranten im privaten Rahmen treffen und sie beim Erlernen der Sprache unterstützen. Dazu zählt auch das Festigen von Fachvokabular für bestimmte Berufsgruppen: beispielsweise unterstützen Ärztinnen und Ärzte sowie Diplomingenieurinnen und -ingenieure die Migrantinnen und Migranten bei der Nostrifizierung ihrer Fachausbildung in Österreich. Bisher konnten über 70 Migrantinnen und Migranten auf dem Arbeitsmarkt Beschäftigung finden. Zur Integration gehört auch das Kennenlernen des Landes und der Kultur. AMAL organisiert Kulturfahrten zu bedeutenden Kulturstätten, um den Schatz der österreichischen Kultur zu erleben. Auch das gemeinsame Feiern von Festen, der Austausch von Werten und Bräuchen zählt dazu. Das Kernanliegen von AMAL ist die Förderung aller Mitglieder der Familie. Das Angebot richtet sich an geflüchtete Eltern, junge Erwachsene, Jugendliche und Kinder aus dem Nahen Osten. Ein weiteres zentrales Element von AMAL ist die Begegnung auf Augenhöhe. Menschen sehnen sich danach, nicht als „Migrant/in“ oder „Nummer“ abgestempelt zu werden, sondern als Mensch gesehen und wertgeschätzt zu werden. Daher hatten die Migrantinnen und Migranten auch von Anfang an die Möglichkeit, aktiv an den Angeboten von AMAL mitzuarbeiten.

Seit der Vereinsgründung sind Syrerinnen und Syrer auch im Vorstand vertreten, um ihre Ideen und die Anliegen ihrer Community einzubringen.

$\mathrm{Zu}$ den weiteren Angeboten von AMAL zählen Elternbildungsseminare, um die Eltern in der Erziehungsrolle in ihrer neuen Heimat zu unterstützen, Bewerbungsworkshops, Sommeraktivitäten für Jugendliche und Kinder, interkulturelle Koch- und Backrunden und die Unterstützung bei rechtlichen, schulischen und gesundheitlichen Themen.

\section{Wesentliche Herausforderungen der Coronakrise und der Lockdowns}

Die Situation der AMAL-Teilnehmerinnen und -Teilnehmer war bis zum Ausbruch der Pandemie stabil: Nach den ersten schwierigen Jahren des Erlernens der Sprache, der 
beruflichen (Neu-) Orientierung und des Aufbaus eines neuen sozialen Umfelds kam eine unerwartete Krise; die Mitglieder waren gezwungen, nochmals eine Herausforderung anzunehmen.

Die Herausforderungen sind im Wesentlichen Jobverlust bzw. Kurzarbeit. Die bereits schwierige finanzielle Situation der Familien hat sich dadurch noch mehr verschlechtert. Zahlreiche Mitglieder waren im März 2020 noch auf Jobsuche. Für sie gibt es jetzt keine Hoffnung, dass sich ihre Situation in den kommenden Monaten verbessern wird. Vor allem für Männer über 50 Jahre mit niedrigen Qualifikationen aus den Herkunftsländern sind die Chancen auf eine baldige Anstellung gering. Für Familien war vor allem der erste Lockdown eine Herausforderung: das Zusammenleben auf engem Raum, das Homeschooling der Kinder (vor allem das Erlernen von neuen Inhalten in Deutsch und das Verbessern der Aufgaben, fehlende digitale Infrastruktur) und der Ausfall von Freizeitaktivitäten.

\section{Nutzung von bestehenden Angeboten des Vereins durch die Mitglieder}

Die Sprachkonversationsrunden wurden seit dem ersten Lockdown auf OnlinePlattformen bzw. telefonisch durchgeführt. Das ist eine wichtige Unterstützung, vor allem für nichtberufstätige Frauen, die sonst die deutsche Sprache gar nicht praktizieren und so in ihrem Erfolg wieder zurückfallen würden. Während des Sommers 2020 waren auch Kulturfahrten mit einer kleineren Anzahl von Mitgliedern möglich. Dieses Angebot wurde sehr gut genutzt. Besonders für Familien und ältere Ehepaare waren diese Kulturfahrten eine willkommene Abwechslung und eine Chance, mit Österreicherinnen und Österreichern in Kontakt zu sein. Auch die beliebten Sommerwochen für Mädchen und Buben konnten dank des entsprechendem Corona-Hygienekonzepts stattfinden. Für viele der teilnehmenden Kinder waren diese Sommerwochen die einzigen Ferientage mit sinnvollem und gleichzeitig lustigem Programm in Gemeinschaft, Abenteuer und neuen Erfahrungen.

\section{Ausbau von Unterstützungsangeboten}

Aufgrund der veränderten Umstände musste auch AMAL sein Programm flexibel anpassen. Viele Angebote, wie z.B. Workshops oder Kulturfeste, waren nicht möglich. Zahlreiche neue Angebote sind dazugekommen:

1. Wöchentliche Newsletter: Für AMAL ist die Motivation und Inspiration der Teilnehmer/innen in herausfordernden Zeiten ein Herzensanliegen. Daher wurde seit Beginn des ersten Lockdowns ein Newsletter eingeführt. Tipps zum Praktizieren der deutschen Sprache, für gemeinsame Familienaktivitäten und das bewusste Gestalten der Zeit sowie ermutigende Kurzvideos sind Hauptthemen dieser Newsletter. Einige Migrantinnen und Migranten erstellten selbst Kurzvideos 
für AMAL, in denen sie erzählten, wie sie die Krise bewältigen, was ihnen in dieser schwierigen Zeit Halt gibt usw.

2. Telefonische Befragung der Wünsche und Bedürfnisse der AMAL-Teilnehmenden: Es galt zu eruieren, welche Themen die AMAL-Teilnehmerinnen und -Teilnehmer während dieser herausfordernden Zeit des Lockdowns beschäftigten. Im April 2020 haben wir dazu eine telefonische Befragung gestartet. Die Ergebnisse dieser Umfrage waren in Stichworten: sprachliche Schwierigkeiten bei nichtberufstätigen Frauen; der verminderte Selbstwert bei Frauen und Männern, die schon längere Zeit arbeitslos sind bzw. im Zuge der Coronakrise ihren Arbeitsplatz verloren haben; die Herausforderungen bei der Jobsuche sowie der eingeschränkte Kontakt zu Österreicherinnen und Österreichern. AMAL hat das Angebot der Sprachkonversation angepasst und digital bzw. telefonisch weitergeführt. Einige ehrenamtliche Mitarbeiterinnen und Mitarbeiter wirkten bei der Jobvermittlung aktiv mit. Sie unterstützten bei der Erstellung und Verbesserung von Lebensläufen, machten auf Jobinserate aufmerksam und coachten für Bewerbungsinterviews.

3. Nachhilfe in Coronazeiten: Kinder aus Migrant/inn/enfamilien hatten schon vor der Coronakrise oftmals schulische Schwierigkeiten. Die Lerndefizite wurden während des Homeschoolings des ersten Lockdowns noch größer. AMAL hat deshalb als unterstützendes Angebot die Corona-Nachhilfe eingeführt. Die Schülerinnen und Schüler erhalten vor allem in Deutsch und Englisch wöchentliche Nachhilfestunden von Studentinnen und Studenten, um die Rückstände aufzuholen. Dies war je nach Pandemiegeschehen vor Ort möglich oder online. Dieses Angebot ist ein Schlüsselfaktor in diesem Schuljahr, in dem der Präsenzunterricht immer wieder durch Fernunterricht ersetzt wurde.

4. Vermittlung von ehrenamtlichen Tätigkeiten: Menschen, die ihr ganzes Leben lang berufstätig waren, möchten arbeiten. Sie sind dankbar für die Unterstützungsleistungen, die sie in Österreich unverdient bekommen und möchten im Gegenzug einen aktiven Beitrag in der Gesellschaft leisten. AMAL hat deshalb im Herbst 2020 das Projekt der Vermittlung von ehrenamtlichen Tätigkeiten ins Leben gerufen. Wir versuchen, arbeitslose Männer ab 55 Jahren, die in Österreich kaum mehr die Möglichkeit haben, eine berufliche Beschäftigung zu finden, an gemeinnützige Einrichtungen zu vermitteln, wo sie sich einbringen können. Entscheidend für den Erfolg dieses Projektes sind die jeweiligen Bestimmungen, denn z.B. konnten während der Lockdowns ehrenamtliche Tätigkeiten oftmals nicht stattfinden. 


\section{Das Arabisch-österreichische HaUs für KUnST UND KULTUR}

Das Arabisch-österreichische Haus für Kunst und Kultur wurde im Jahr 2014 gegründet und hat mehr als 50 Mitglieder. Es ist eine Institution (organisiert als Verein), die es sich zur Aufgabe gemacht hat, die arabische Kultur zu präsentieren und zu vermitteln. Um dies zu erreichen werden nachstehende Ziele verfolgt:

\section{- Bücherei/Bibliothek}

Einer der Grundpfeiler des Hauses ist es, die aktuellen und prominentesten Neuerungen auf den Gebieten Literatur, Kunst und Wissenschaften aus der arabischen Welt zur Verfügung zu stellen und diese über Büchereien verleihbar zu machen. Diskussionsrunden in den Bibliotheken sollen zum Dialog mit den Autor/inn/en, Verfasser/ inne/n und den arabischen Schriftsteller/inne/n einladen. Dies impliziert, dass diese Bücher in der Bibliothek auch in der deutschen und weiteren europäischen Sprachen zur Verfügung gestellt werden.

\section{- Theater und arabisches Kino}

Das Haus bietet in seinem Gebäude Räumlichkeiten zur Aufführung von Filmen und kurzen Theaterstücken an sowie die Möglichkeit anschließender Diskussionsrunden.

\section{- Musikhalle/Auditorium}

Eine Musik-Bibliothek soll die Werke arabischer Musiker/innen zum Anhören bereitstellen. $\mathrm{Zu}$ den Zielen des Hauses gehören auch Angebote in Bezug auf das Erlernen und das Studium der arabischen Musik.

\section{- Ausstellungsraum für bildende Kunst}

Geplant ist ein Ausstellungsraum, der sowohl arabischen als auch österreichischen Künstler/inne/n Präsentationsflächen bietet. Es sollen auch Räumlichkeiten für eine Dauerausstellung arabischer Künstler/innen, die hier in Österreich leben, bereitgestellt werden. Ein weiteres Ziel ist die Vermittlung von Kenntnissen der arabischen Kalligrafie und das Anbieten von Kursen zum Erlernen derselben.

\section{- $\underline{\text { Studium, Forschung und Publikation }}$}

Das Haus verfügt auch über eine Abteilung, die den in Österreich sesshaften arabischen Bürger/inne/n die Weiterbildung mit arabischem, kulturellem Hintergrund in ihrer Sprache ermöglicht. Dieses Angebot soll jedoch auch in deutscher Sprache erfolgen, um 
insbesondere den interessierten Österreicher/inne/n, aber auch weiteren deutschsprachigen und europäischen Mitbürger/inne/n einen Zugang zu bieten.

Diese Institution macht es sich weiters zur Aufgabe, die Berichterstattung über den arabischen Raum in der hiesigen Medienlandschaft zu beobachten und im Bedarfsfall korrigierend zu agieren, um Missverständnisse aufzuklären. Es werden Kooperationsmöglichkeiten und Kontakte zu Verlagshäusern, Kulturinstitutionen sowie zu den österreichischen und weiteren europäischen Medien gesucht, um arabische Sichtweisen zu aktuellen Geschehnissen darlegen zu können. Es ist auch beabsichtigt eine eigene Druckerei zu installieren, in der alle notwendigen Drucksachen der einzelnen Abteilungen des Hauses hergestellt werden können.

Unter der Leitung des Hauses sollen Druckwerke (Zeitungen, Magazine usw.), Internet, Radio- und Fernsehsender als Sprachrohr dienen. Dies sind Planungen auf lange Sicht, die je nach Unterstützung und Förderung, die dem Haus von arabischer oder europäischer Seite zukommen, verwirklicht werden sollen.

\section{Arabische Schule}

$\mathrm{Zu}$ den Abteilungen gehört auch die arabische Schule für arabische und österreichische Mitbürger/innen. Diese steht sowohl Anfänger/inne/n als auch Fortgeschrittenen offen, die an Instituten die arabische Sprache gelernt haben und ihre Kenntnisse vertiefen möchten.

\section{Arabisches Café}

Das Haus möchte auch einen Ort mit arabischem Flair und Küche bieten, an dem man sich gerne trifft und einander kennenlernen kann. Es soll in erster Linie ein Künstlertreff werden, an dem man gerne verweilt.

Um die o.a. Pläne umzusetzen und die angepeilten Ziele zu erreichen, müssen folgende Punkte erfüllt werden:

○ die entsprechenden Räumlichkeiten finden, die Kosten berechnen und die Finanzierung planen: Hierzu sollten entsprechend erfahrene Personen auf diesem Gebiet sowohl von österreichischer als auch arabischer Seite hinzugezogen werden.

- mit Spezialist/inn/en die beste Umsetzungsform erörtern (Verein, $\mathrm{GmbH}$ etc.) und die Auswahl der damit beauftragten Gruppe zur Durchführung und Ernennung eines Kuratoriums.

- die schrittweise Einführung und Bekanntmachung des Projektes und entsprechende Pressearbeit: dies bereits vor der Fertigstellung unter Einbindung der arabischen Foren und Vereine hier im Land. Auch die Einbindung der Kulturzentren der arabischen Botschaften hier in Wien muss berücksichtigt werden. 
$\circ$ der Austausch mit den verschiedenen arabischen Forschungszentren, um eine Kooperation zur Sicherung der Projekte des Hauses zu gewährleisten.

○ der Aufbau von Beziehungen zu den unterschiedlichen österreichischen Kunstund Kulturvereinen gehört ebenso zu den Aufgaben, um wichtige Anregungen aus deren Erfahrungen und Vorgangsweisen zu schöpfen und den Erfolg der geplanten Projekte im Haus zu sichern.

- Ausbau von Unterstützungsangeboten (Ist etwas Neues hinzugekommen? z.B. neue Facebook-Gruppe, neue Themen etc.)

Die Covid-19-Pandemie und die Lockdowns waren besonders schwierig, da für unsere Mitglieder das wöchentliche Treffen sehr wichtig ist. Wir haben deshalb neue Facebook-Gruppen gegründet und regelmäßige Treffen über Zoom organisiert.

Bei den zahlreichen Zoom-Meetings war besonders das Frauen-Komitee sehr aktiv. Unter anderem wurden und werden folgende Themen in den Zoom-Meetings behandelt:

\section{Anstellung oder Selbständigkeit?}

In Zeiten einer weltweiten Gesundheits- und Wirtschaftskrise und der höchsten Arbeitslosigkeit in Österreich in der Zweiten Republik verursacht durch Covid-19 haben sich viele Mitglieder und Interessierte diese Frage gestellt. Im Rahmen dieses Online-Forums wird darüber informiert.

\section{Bildung und Ausbildung in Österreich - Kinder auf dem Bildungsweg begleiten:}

Diese Veranstaltung richtete sich an Eltern und Erziehungsberechtigte und behandelte folgende Fragen: Wie funktioniert das Bildungs- und Ausbildungssystem in Österreich? Wie wählt man den richtigen Bildungsweg aus? Welche Rolle habe ich als Elternteil oder Erziehungsberechtige/r für den Bildungsweg meines Kindes?

\section{Lernunterstützungen für Kinder:}

Diese erfolgen in Kooperation mit der Culture School. Ziel ist es, ein positives Miteinander in den Schulen zu gestalten und den Lernerfolg aller Schüler/innen damit zu fördern. Diese Veranstaltungen werden für die Eltern und Erziehungsberechtigten in den Arabisch und Farsi sprechenden Communities organisiert.

\section{Förderung für Schüler/innen mit erhöhtem Förderbedarf:}

Welche speziellen Bedürfnisse haben diese? 


\section{Das Entwicklungspotential der Frau:}

Dabei werden folgende Themen diskutiert: Stärken und Entwicklungsfähigkeiten erkennen und diese umsetzen; Methoden erlernen, um die eigene Kraft und vorhandene Potentiale zu stärken.

\section{Lesungen von Gedichten sowie musikalische Darbietungen (Arabisch/Deutsch).}

Es gibt auch verschiedene Facebook-Seiten, z.B. Kultur-, Musik- oder FilmSeiten, auf denen täglich verschiedene Links zu aktuellen Online-Aktivitäten gepostet werden.

Natürlich wünschen wir uns mehr finanzielle Unterstützung für unsere Projekte, da die Einreichung an die verschiedenen Fördergeber meistens sehr kompliziert ist. 


\section{Vorstellung Des SPORT- UND KuLTURVEREINS „NEUER START“}

Der Verein „NEUER START“ wurde im Jahr 2010 als Initiative für die Unterstützung geflüchteter Menschen sowie zur Herstellung von Kontakten zwischen Geflüchteten untereinander, aber auch zur österreichischen Bevölkerung ins Leben gerufen und sodann im Jahr 2012 mit dem Namen „Afghanische Jugendliche NEUER START“ in Österreich als offizieller Verein bei den zuständigen Vereinsbehörden gemeldet. Er ist ein spendenfinanzierter Wiener Verein, welcher Geflüchtete im Integrationsprozess unterstützt.

Im Herkunftsländervergleich ist Afghanistan das Land, aus dem die meisten unbegleiteten minderjährigen Flüchtlinge kommen. Der Weg nach Europa ist ein einsamer, welcher oftmals von schlimmen Erfahrungen und vielen Gefahren geprägt ist. Mit der Zeit finden sich die Geflüchteten meist gut in Österreich zurecht, sind integriert, können die Sprache, verfügen über eine Ausbildung und einen Arbeitsplatz und respektieren die österreichischen Gesetze und Regeln. Um an diesen Punkt zu gelangen, sind die Geflüchteten jedoch üblicherweise mit einer Reihe von Problemen konfrontiert. Die Unsicherheit über das Bleiberecht und die Unkenntnis über das Asylverfahren sowie über bürokratische Abläufe und die fehlenden Sprachkenntnisse sind nur der Beginn der vielen Hürden, die von den Geflüchteten überwunden werden müssen. Hinzu kommen zudem oftmals traumatische Erfahrungen von Gewalt, Krieg und Flucht. Sind die anfänglichen Probleme gelöst, kommen allerhand neue Herausforderungen auf die Geflüchteten zu, wie zum Beispiel das Finden einer Wohnung, einer Ausbildungsstelle oder einer Arbeit. Banal anmutende, alltägliche Tätigkeiten wie das Eröffnen eines Kontos oder das Abschließen eines Handyvertrags gestalten sich als schwierig. Um einen möglichst reibungslosen Integrationsprozess zu ermöglichen, wurde der Verein ,Neuer Start“6 für afghanische Jugendliche ins Leben gerufen.

Das Spektrum an Aktivitäten wird seitdem stetig erweitert. Diese beinhalten unter anderem das jährlich veranstaltete Integrationsfestival „Von Kabul bis Wien“ für MigrantInnen und ÖsterreicherInnen, Workshops an österreichischen Schulen in Zusammenarbeit mit der Asylkoordination, mit dem Kardinal-König-Haus und dem UNHCR Österreich und anderen Organisationen sowie Workshops zum Thema „Umgang mit Geld und Banken“ gemeinsam mit der „Zweiten Sparkasse“ und „lobby.16“ usw. Auch die Berufsorientierung zählt zu unserem Aufgabengebiet. Hierbei liefern wir Aufklärung über das österreichische Bildungssystem und unterstützen beim Zusammenstellen von Bewerbungsunterlagen. Auch beim Erwerb der nötigen Sprachkenntnisse bieten wir Unterstützung an: erstens durch das Vermitteln von Deutschkursen und zweitens durch den Klub der Muttersprache, wo die Menschen in ihrer eigenen Sprache, Dari und Paschto, alphabetisiert werden und so rascher Deutsch erlernen können. 
Eines unserer zentralen Ziele ist die Sensibilisierung und Bewusstseinsbildung der österreichischen Bevölkerung, d.h. Verständnis für die Situation der Flüchtlinge, für ihre Ängste, für ihre Werte, ihre Religion, zu fördern. Dazu haben wir gemeinsam mit der Internationalen Organisation für Migration (IOM) und dem UN-Flüchtlingshochkommissariat (UNHCR) eine Simulationskonferenz für Wiener SchülerInnen zum Thema „Integration von jungen Flüchtlingen in Schulen“ ins Leben gerufen. Weiters haben wir schon mehrmals gemeinsam mit dem UNHCR Schulworkshops und Gesprächsrunden im Rahmen des „Langen Tags der Flucht“ durchgeführt und bieten Vorträge zum Thema „Lebenswelt afghanischer Geflüchteter“ an, in denen das Geburtsland der Menschen sowie die „neue Heimat“ thematisiert werden.

Ergänzend ist zu sagen, dass sich unsere Arbeit keinesfalls nur auf afghanische Flüchtlinge beschränkt. Im Gegenteil, wir versuchen Geflüchtete aus allen Ländern in unsere Projekte einzubinden. Unser Ziel ist es, den interkulturellen Dialog zu stärken sowie die Chancengleichheit für Jugendliche mit Migrationshintergrund zu ermöglichen und ihnen zu einem erfolgreichen neuen Start zu verhelfen. Wir haben über 70 Mitglieder darunter 8 im Vorstand und 4 im Schiedsgericht.

Der Verein hieß von 28.01.2018 bis 30.05.2020 „Afghanischer Sport- und Kulturverein NEUER START”, aber im Vereinsregisterauszug stand „Afghanischer Sport- und Kultur-Verein NEUER START". Wir haben am 30.05.2020 bei der letzten Generalversammlung beschlossen, dass der Name des Vereins wieder geändert werden sollte. Seit 30.05.2020 trägt er die Bezeichnung ,Sport- und Kulturverein NEUER START”.

\section{Unsere Haltung zum Thema Integration}

Für jeden und jede bedeutet Integration etwas Anderes. Für uns bedeutet sie gegenseitiges Akzeptieren und Respektieren. Integration kann nicht einseitig sein und von heute auf morgen gelingen, sie muss von beiden Seiten ermöglicht werden, von den Geflüchteten, aber auch von ÖsterreicherInnen. Beide Seiten müssen sich bemühen und aufeinander zugehen. Dabei sind Sprache, Kultur und die Regeln sehr wichtig, die in der Gesellschaft bestehen, in der man neu ist, und die man Schritt für Schritt kennenlernen muss. Wichtig ist unserer Ansicht nach, dass man über die Grundgesetze der Verfassung und über die Traditionen des neuen Landes Bescheid weiß. All das hilft, um sich wohlzufühlen, ohne dabei seine eigenen Wurzeln vergessen zu müssen. Die Schlüssel zur Integration sind für uns Freundschaft, Offenheit und Bildung. In einer Freundschaft ist es egal, woher du kommst oder wie du aussiehst - wichtig ist ja nur, wer du bist! Ohne Offenheit entwickelt sich eine Person, aber auch eine Gesellschaft nicht weiter. Wir müssen daher aufeinander zugehen, wieder und immer wieder. Und Bildung ist ein wesentlicher Baustein für Integration und für ein selbstbestimmtes Leben. Für all das setzen wir uns im Verein ein.

Mehr über unsere Geschichte: < https://www.neuerstart.at/uber-uns/>

Artikel mit oder über uns: <https://www.neuerstart.at/artikeln-uber-uns/> 


\section{Herausforderungen von COVID-19 und der Lockdowns für uns}

Der Schulunterricht wurde online abgehalten, Amtswege wurden per Telefon oder online erledigt, Informationen über das Virus sowie aktuelle Verhaltensregeln online verbreitet, Arbeitslose mussten sich online beim AMS anmelden.

Wer heute keinen Computer, kein Internet oder kein Smartphone besitzt, ist von der Welt mehr oder weniger abgeschnitten. Mit dem Gebot zu Hause zu bleiben, waren wir alle auf TV- und Online- sowie vereinzelt auf Telefondienste als Informationsquellen angewiesen.

Was aber, wenn die Deutschkenntnisse (noch) nicht ausreichten, um den offiziellen österreichischen TV- und Zeitungsnachrichten zu folgen? Jede/r, der/die schon einmal eine Fremdsprache erlernt hat, weiß, wie schwierig und problematisch es ist, ein höheres Sprachniveau, wie es z. B. von PolitikerInnen oder ExpertInnen in Pressekonferenzen oder TV-Debatten angewandt wird, zu verstehen, selbst wenn man der Alltagssprache mächtig ist.

Wir haben versucht, für unsere Mitglieder und die Community schriftliche Informationen in ihrer Muttersprache zum Downloaden auf ihren Websites oder Social Media zu verbreiten. Wir waren darüber hinaus telefonisch für Beratungen und Informationen erreichbar.

Weiters konnten Digitalisierungskurse in der Sprache Dari/Farsi für Dari/Farsi Sprechende seitens des Vereins Neuer Start ehrenamtlich in Kooperation mit dem Verein „Fivestones“ im CORE-Zentrum sehr erfolgreich angeboten werden. Wir würden gerne auch in anderen Sprachen flächendeckend in Österreich solche Angebote erbringen: Dies ist leider auf ehrenamtlicher Basis nicht in dem Maß möglich, wie wir uns dies wünschen würden.

Wir haben zur jetzigen Situation genaue und seriöse Informationen auf Dari/Farsi, Paschto und Deutsch auf unserer Dari/Farsi-Homepage (<https://www.neuerstart.at/>) hochgeladen, die zur weiteren Verbreitung dienen.

Wir bemühen uns, etwaige Änderungen oder Informationen aus seriösen Quellen zum Coronavirus sofort auf Dari/Farsi und Paschto upgedatet anzubieten, damit sich Geflüchtete in dieser schwierigen Zeit orientieren und richtig verhalten können.

Wir arbeiten seit 2010 ehrenamtlich. Wir würden sehr gerne unsere Projekte und Aktivitäten ( $<$ https://www.neuerstart.at/category/projekte-und-aktivitaten/ $>$ ) weiterentwickeln, was jedoch ohne Förderungen und Unterstützungen sehr schwierig und teilweise demotivierend ist. Wir wünschen uns daher, dass MigrantInnenvereine hinsichtlich der Förderungen nicht wie einheimische Vereinen behandelt werden, weil Deutsch nicht unsere Muttersprache ist und wir daher mit größeren Herausforderungen konfrontiert sind. 